$\xi=2$ 줄

\title{
The harmless imitator of OSCC: TUGSE - traumatic ulcerative granuloma with stromal eosinophilia
}

\author{
Anuradha Pai, Karthik D Yadav *, Ramakrishna T, Abhishek B \\ The Oxford Dental College, 10th Milestone, Bommanahalli, Hosur Road, Bangalore- 560 102, India \\ *Corresponding author E-mail: karthikyadavd@gmail.com
}

\begin{abstract} Carcinoma; Self-Limiting

\section{Introduction}

Traumatic ulcerative granuloma with eosinophilic stroma (TUGSE) also known as traumatic eosinophilic granuloma or eosinophilic ulcer of the oral mucosa is a rare and reactive lesion with a benign clinical course. It is an uncommon, self-limiting lesion, of inflammatory nature preferentially found on the lateral and ventral surface of the tongue, but can also occur in the lip, palate, mucobuccal fold and gingiva. Despite the fact that the fact that the pathogenesis is not clear, an essential role has been related to trauma (Burket LW 2015, p88-89, Neville BW et al 2004 p255258).
\end{abstract}

Traumatic Ulcerative Granuloma with Stromal Eosinophillia is a rare, benign, self-limiting and asymptomatic lesion that shows a spontaneous regression. Its etiopathogenesis is an enigma, but trauma seems to play a role for the occurrence of this lesion. Clinically it manifests as an isolated long standing lesion predominantly located in the tongue, demonstrating raised \& indurated margins and mimicking oral squamous-cell carcinoma. Microscopically it is characterized by an inflammatory infiltrate rich in eosinophils. Since these lesions show delayed healing, treatment becomes necessary, but in certain cases, healing occurs by excision of the ulcer alone during the biopsy.

Keywords: Traumatic Ulcerative Granuloma with Stromal Eosinophilia; Oral Squamous-Cell Carcinoma; Mucoepidermoid Carcinoma; Adenoid Cystic

\section{Case report}

A 68-year-old male visited the department of oral medicine and radiology, bangalore with the chief complaint of ulcer on the tip of tonguesince 16 days. On eliciting history patient revealed that the lesion initiated as an ulcer which gradually changed to a small mass, which was tender and associated with difficulty while having food. The pain was felt more in the morning. He also gives a history of ulcers in the oral cavity since 6 months which usually heals by itself within a week without any medication.

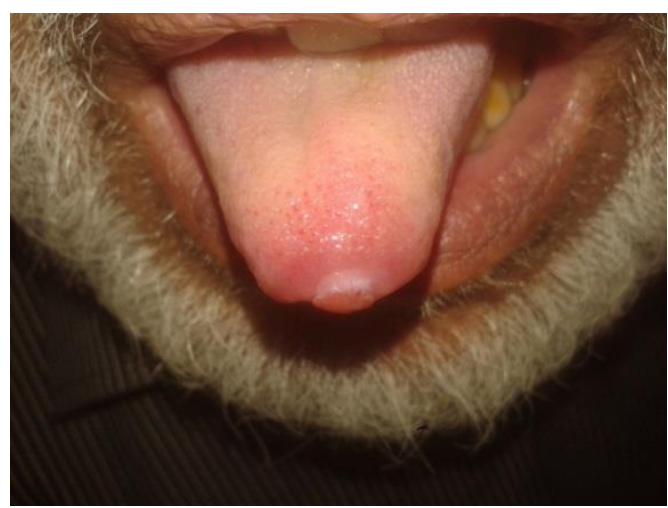

Fig. 1: Showing A Well-Defined Solitary Exophytic Growth was noticed at the Tip of the Tongue.

On eliciting medical history, patient revealed that he had undergone angioplasty before 6 months and was on medication (Aspirin $75 \mathrm{mg}$ once daily since 6 months). On clinical examination a welldefined solitary exophytic growth was noticed at the tip of the tongue which was sessile, measuring about $0.7 \mathrm{~cm}$ with irregular surface and everted margins with erythematous borders. On palpation it was tender and firm in consistency. Based on the above findings a provisional diagnosis of traumatic fibroma and a differential diagnosis of oral squamous cell carcinoma was given.

Enteroplasty of the maxillary and mandibular anterior teeth of both arches was done and night guard was provided as a symptomatic treatment. The lesion was under observation for two weeks to check for any signs of healing but as there was no significant changes were observed, we planned for the excision of the lesion. Under local anesthesia, excisional biopsy from the lesion was done with a $0.5 \mathrm{~cm}$ margin from the lesion by electrocautery, and the specimen was submitted for histopathological examination. Section showed parakeratinized stratified squamous epithelium with areas of ulceration covered by fibrinopurulent membrane. The connective tissue shows numerous engorged capillaries lined 
by epithelial cells with inflammatory infiltrate consisting of eosinophils, lymphocytes and plasma cells, suggestive of traumatic ulcerative granuloma with eosinophilic stroma.

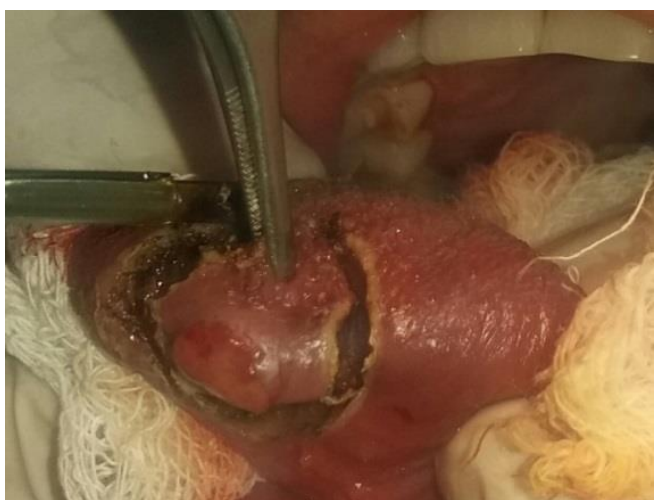

Fig. 2: Showing the Excision of Lesion by Electro cautery.

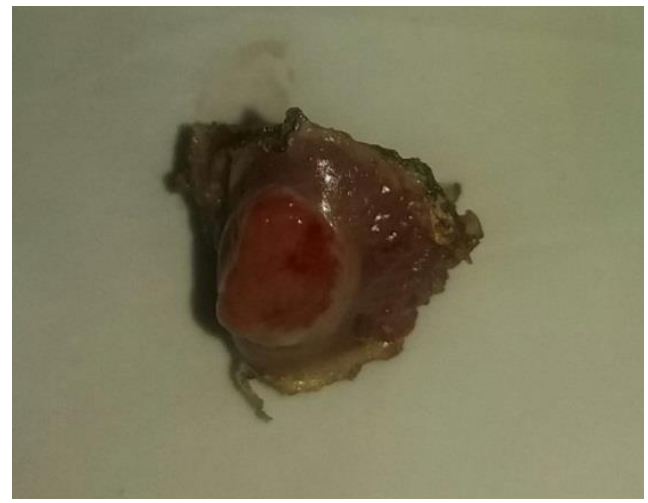

Fig. 3: Shows the Excised Specimen.

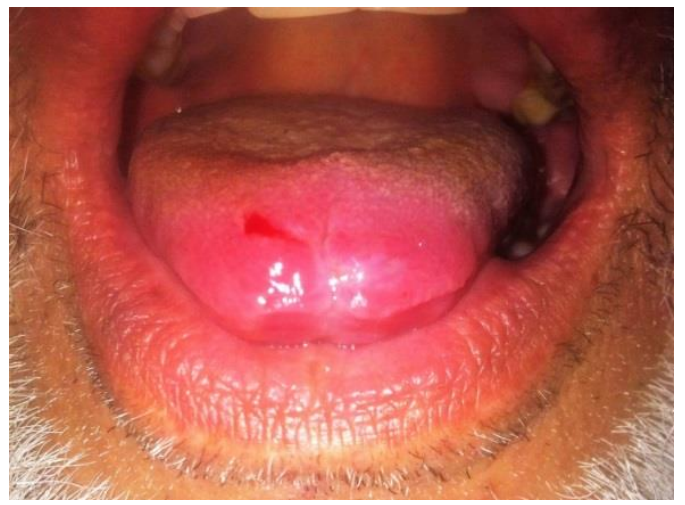

Fig. 4: Showing Healing Lesion after 2 Weeks.

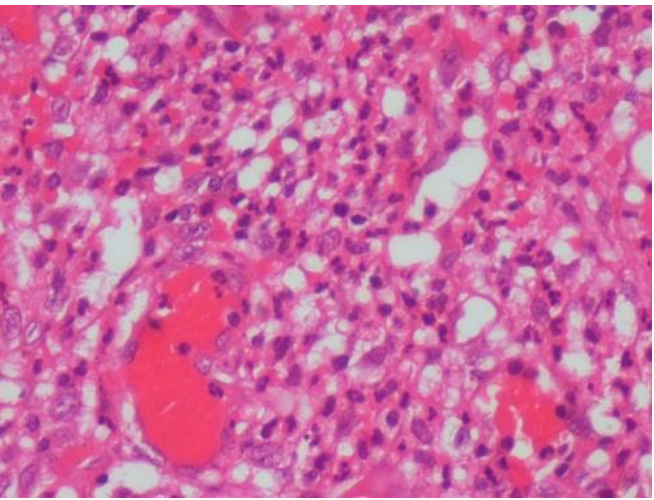

Fig. 5: Under 40x Microscopic Magnification the Section Shows Numerous Eosinophils with Lymphocytes and Proliferating Endothelial Cells Surrounding the Capillary and Edematous Areas.

\section{Discussion}

Traumatic ulcerative granuloma with eosinophilic stroma (TUGSE) was initially depicted in adults by Popoff in 1956. Since then, different names such as TGSE (Elzay, 1983; Hirshberg et al, 2006); traumatic eosinophilic granuloma of the tongue (Ficarra et al, 1997; Alobeid et al, 2004) and eosinophilic ulcer under the tongue, or the oral mucosa. ) has been used to describe this lesion leading to further confusion (Raviteja YS et al).

TUGSE is also called by other names, including eosinophilic granuloma, eosinophilic ulcer, Riga Fede disease (in infants and children) and atypical histiocytic granuloma (Chavan SS et al). TUGSE can show a slight male predominance and is more evident to the age of 60-70 years anywhere in the oral cavity with the most common sites being the ventral surface of the tongue, buccal mucosa, vestibule and floor at the mouth. Although it usually follows some form of trauma, most patients do not have a history of trauma, and the suggested pathogenesis is ulceration resulting from some form of trauma, which permits the ingress of microorganisms, toxins, or foreign protein into the connective tissue (Chavan SS et al).

Most commonly, the lesion appears as a self-healing solitary ulcer with rolled up or indurated margins of weeks to month's duration, but it can also present as multiple recurrent self-healing ulcers. Individual lesions appear as areas of erythema surrounding central area of yellow fibrinopurulent membrane, which can be removed. Sometimes a rolled white border of hyperkeratosis is seen immediately adjacent to the area of ulceration. Because of such features, it is likely to be confused for oral malignancy such as squamouscell carcinoma (Sivapathasundharam B et al).

A Spectrum of conditions which manifest themselves as a single lesion should be considered in the differential diagnosis such as oral squamous-cell carcinoma, haematological neoplasms and salivary gland malignancies (mucoepidermoid and adenoid cystic carcinoma). Solitary ulcers which persist for more than 2 weeks after treatment without signs of evident healing must be taken seriously, and a biopsy becomes mandatory(Raviteja YS et al).

Histologically, it shows granulation tissue in the ulcer bed that supports mixed inflammatory cell infiltrate of lymphocytes, histiocytes, neutrophils, and occasionally plasma cells. Inflammatory infiltrate to extend into the deeper structures and exhibits sheets of lymphocytes and histiocytes inter mixed with eosinophils. Vascular connective tissue, deep to the ulceration may become hyper plastic and cause the adjacent surface raising. (Raviteja YS et al)

Wait-and-watch should be followed as an approach for healing, which usually occurs within 1 month, but may sometimes take as long as 8 months $^{3}$. Antibiotics, topical, intralesional and/or systemic corticosteroids, have been tried in longstanding cases. An incisional biopsy is often required for definitive diagnosis when no evidence of spontaneous healing is observed at 1-month follow up. Recurrence has been reported rarely. Curettage, cryosurgery is also helpful, but surgical excision is the most commonly cited treatment procedure among the different therapies used (Raviteja YS et al).

Diagnosis of TUGSE is based on clinical and histologic features that include the presence of an ulcer with a polymorphic inflammatory infiltrate including lymphocytes and abundant eosinophils, extending into deeper tissues, admixed with scattered large atypical histiocyte-like cells with large vesicular nuclei and prominent nucleoli and often with mitotic figures (Chavan SS et al).

\section{Conclusion}

Traumatic granuloma is a benign reaction of the oral mucosa with trauma as the contributing factor. Recognition is important because it often mimics oral cancer. In most of the cases, the lesions heal spontaneously so there is no need for surgery. Clinical, histopathological and follow-up data are crucial to achieve a correct diagnosis in the difficult and uncommon cases and to avoid possible over treatment (Regezi JA et al). 


\section{References}

[1] Burket LW, Greenberg MS, Glick M, Ship JA. Burkets Oral Medicine. 12ed: BC Decker Inc; 2015, p 88-89.

[2] Neville BW, Damm DD, Allen CM, Bouquot JE. Text Book of Oral and Maxillo Facial Pathology. 2nd Ed., Elsevier, New Delhi; 2004, p 255-258.

[3] Raviteja YS, Kundana I, Triveni T. Eosinophilic Ulcer of the Oral Mucosa. Int J Dent Med Res 2015; 1(5):112-115.

[4] Chavan SS, Reddy P. Traumatic ulcerative eosinophillic granuloma with stromal eosinophilia of tongue. South Asian J Cancer. 2013 Jul-Sep; 2(3): 144. https://doi.org/10.4103/2278-330X.114128.

[5] Sivapathasundharam B, Lavanya S. Traumatic ulcerative granuloma with stromal eosinophilia (TUGSE). J Oral MaxillofacPathol 2005; 9:30-3. https://doi.org/10.4103/0973-029X.39058.

[6] Regezi JA, Zarbo RJ, Daniels TE, Greenspan JS. Oral traumatic granuloma: Characterization of the cellular infiltrate. Oral Surg Oral Med Oral Pathol. 1993:75: 112. https://doi.org/10.1016/00304220(93)90430-C. 